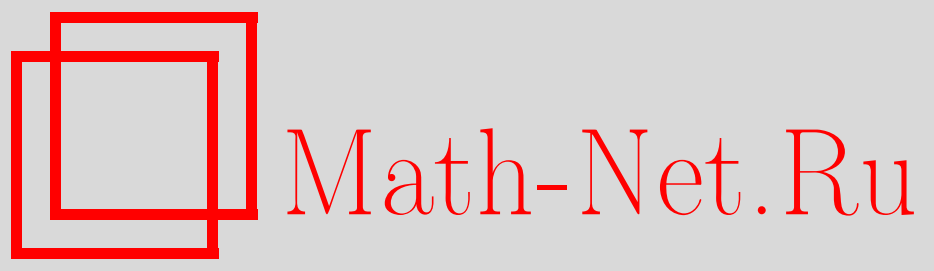

С. Ю. Вернов, Построение точных решений в двухполевых космологических моделях, ТМФ, 2008, том 155, номер 1, 47-61

DOI: https://doi.org/10.4213/tmf6192

Использование Общероссийского математического портала Math-Net.Ru подразумевает, что вы прочитали и согласны с пользовательским соглашением http://www.mathnet.ru/rus/agreement

Параметры загрузки:

IP: 34.239 .49 .27

26 апреля 2023 г., 03:55:44

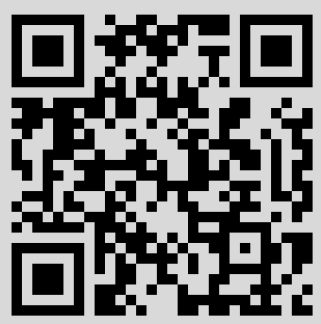




\title{
ПОСТРОЕНИЕ ТОЧНЫХ РЕШЕНИЙ В ДВУХПОЛЕВЫХ КОСМОЛОГИЧЕСКИХ МОДЕЛЯХ
}

\begin{abstract}
Построена модель темной энергии с фантомным скалярным полем, обычным скалярным полем и полиномиальным потенциалом, происходящим из полевой теории струн. Найдено двухпараметрическое множество точных решений уравнений Фридмана. Построен потенциал, удовлетворяющий полученным из теории струн условиям и такой, что некоторые точные решения соответствуют при больших временах параметру состояния $w_{\mathrm{DE}}>-1$, тогда как другие соответствуют $w_{\mathrm{DE}}<-1$. Показана эффективность метода суперпотенциала при поиске новых точных решений.
\end{abstract}

Ключевые слова: полевая теория струн, космология, фантом, темная энергия, точное решение, метод суперпотенциала.

\section{1. ВВЕДЕНИЕ}

Одним из наиболее важных недавних результатов в космологии является заключение о том, что комбинированный анализ данных, полученных при изучении сверхновых типа Іа, галактических кластеров и на эксперименте WMAP (Wilkinson Microwave Anisotropy Probe), убедительно показывает ускоренное расширение Вселенной [1], [2].

Космологическое ускорение указывает на то, что в настоящее время во Вселенной доминирует равномерно распределенная медленно изменяющаяся космическая жидкость с отрицательным давлением, так называемая темная энергия [3]-[8]. Для спецификации различных типов космической жидкости обычно используется феноменологическое соотношение между давлением (лагранжевой плотностью) $p$ и плотностью энергии $\varrho$ каждой из компонент жидкости $p=w \varrho$. Функция $w$ называется параметром состояния. Современные эксперименты [1]-[4] свидетельствуют о том, что Вселенная является пространственно плоской и в настоящее время параметр состояния темной энергии близок к -1 : $w_{\mathrm{DE}}=-1 \pm 0.2$. Параметр состояния

* Научно-исследовательский институт ядерной физики им. Д. В. Скобельцына, Московский государственный университет им. М. В. Ломоносова, Москва, Россия.

E-mail: svernov@theory.sinp.msu.ru 
$w_{\mathrm{DE}} \equiv-1$ соответствует космологической константе. Как отмечено в работе [9], параметр состояния, эволюционирующий от $w_{\mathrm{DE}} \simeq 0$ до $w_{\mathrm{DE}} \leqslant-1$, лучше отвечает экспериментальным данным, чем $w_{\mathrm{DE}} \equiv-1$.

Стандартным способом получения зависящего от времени параметра состояния является включение скалярных полей в космологическую модель. При достаточно общих предположениях в рамках четырехмерной модели с одним скалярным полем может быть реализована только одна из возможностей: или $w_{\mathrm{DE}} \geqslant-1$ (модели квинтэссенции), или $w_{\mathrm{DE}} \leqslant-1$ (фантомные модели) [10]. Двухполевые модели с пересечением барьера космологической константы $w_{\mathrm{DE}}=-1$ известны как квинтом (quintom) модели и включают одно фантомное скалярное поле и одно стандартное скалярное поле. Отметим, что большинство феноменологических моделей, описывающих пересечение барьера космологической константы [11]-[14], содержат либо несколько скалярных полей, либо модифицированную гравитацию.

В настоящее время струнные и D-бранные модели находят космологические приложения, связанные с ускоренным расширением Вселенной. В феноменологических моделях, описывающих случай $w_{\mathrm{DE}}<-1$, все стандартные энергетические условия нарушены и есть проблемы нестабильности как на классическом, так и на квантовом уровне (см. работы [5], [15], [16] и ссылки в них). Возможным способом избежать проблемы нестабильности фантомной модели является ее построение как эффективной модели, возникающей из более фундаментальной теории с нормальным знаком кинетического члена. В частности, если мы рассмотрим модель с высшими производными, например содержащую $\phi e^{-\square} \phi$, то первая нетривиальная аппроксимация дает $\phi e^{-\square} \phi \phi^{2}-\phi \square \phi$, т.е. кинетический член имеет неправильный (духовый) знак. Оказывается, что именно такая возможность появляется в полевой теории струн [17] (см. также работы [14], [16]), а именно в теории фермионной NSR-струны с учетом GSO-сектора [18]. В качестве скалярного поля ф выступает тахион открытой струны. Согласно гипотезе Сена (см. обзоры [19]) данный тахион описывает распад браны, при котором происходит медленный переход в стабильный вакуум, характеризующийся отсутствием состояний открытой струны, т.е. соответствующий состояниям замкнутой струны. Четырехмерная гравитационная модель с фантомным скалярным полем рассматривается как приближение модели теории струн, что дает возможность решить проблему нестабильности.

В данной статье мы рассматриваем струнную гравитационную модель с двумя скалярными полями и полиномиальным потенциалом. Предлагаемая модель является обобщением однополевой космологической модели, предложенной в работе [18]. Первые двухполевые обобщения данной модели [20] обладают однопараметрическими семействами точных решений. Здесь мы строим новую модель с двухпараметрическим семейством точных решений. При больших временах для некоторых значений параметров $w_{\mathrm{DE}}<-1$, тогда как для других $w_{\mathrm{DE}}>-1$. Отметим, что различное поведение $w_{\mathrm{DE}}$ при больших временах соответствует одному и тому же потенциалу и одинаковым асимптотическим условиям для полей. 


\section{2. ДВУХПОЛЕВЫЕ МОДЕЛИ, СВЯЗАННЫЕ С ТЕОРИЕЙ СТРУН}

Рассматривается модель пространственно плоской фридмановской Вселенной с фантомным скалярным полем $\phi$ и стандартным скалярным полем $\xi$. Фантомное поле представляет собой тахион открытой струны, тогда как обычное скалярное поле соответствует тахиону замкнутой струны [17], [20]-[22]. Поскольку происхождение скалярных полей связано с теорией струн, действие содержит характерную массу струны $M_{\mathrm{s}}$ и безразмерную константу взаимодействия открытых струн $g_{o}$ :

$$
S=\int d^{4} x \sqrt{-g}\left(\frac{M_{\mathrm{P}}^{2}}{2 M_{\mathrm{s}}^{2}} R+\frac{1}{g_{o}^{2}}\left(\frac{1}{2} g^{\mu \nu}\left(\partial_{\mu} \phi \partial_{\nu} \phi-\partial_{\mu} \xi \partial_{\nu} \xi\right)-V(\phi, \xi)\right)\right),
$$

где $M_{\mathrm{P}}$ - масса Планка. Фридмановская метрика $g_{\mu \nu}$ является пространственно плоской:

$$
d s^{2}=-d t^{2}+a^{2}(t)\left(d x_{1}^{2}+d x_{2}^{2}+d x_{3}^{2}\right),
$$

где $a(t)$ - масштабный фактор. Координаты $\left(t, x_{i}\right)$ и поля $\phi$ и $\xi$ являются безразмерными. Если скалярные поля зависят только от времени, то уравнения движения имеют следующий вид:

$$
\begin{gathered}
H^{2}=\frac{1}{3 m_{\mathrm{p}}^{2}}\left(-\frac{1}{2} \dot{\phi}^{2}+\frac{1}{2} \dot{\xi}^{2}+V\right), \\
\dot{H}=\frac{1}{2 m_{\mathrm{p}}^{2}}\left(\dot{\phi}^{2}-\dot{\xi}^{2}\right), \\
\ddot{\phi}+3 H \dot{\phi}=\frac{\partial V}{\partial \phi}, \quad \ddot{\xi}+3 H \dot{\xi}=-\frac{\partial V}{\partial \xi} .
\end{gathered}
$$

Для краткости мы используем безразмерный параметр $m_{\mathrm{p}}^{2}=g_{o}^{2} M_{\mathrm{P}}^{2} / M_{\mathrm{s}}^{2}$. Параметр Хаббла $H \equiv \dot{a}(t) / a(t)$, а точка обозначает производную по времени. Отметим, что только три из четырех уравнений (2)-(4) независимы.

Параметр состояния темной энергии $w_{\mathrm{DE}}$ можно выразить через $H$ :

$$
w_{\mathrm{DE}}=-1-\frac{2}{3} \frac{\dot{H}}{H^{2}} .
$$

Пересечение барьера космологической постоянной $w_{\mathrm{DE}}=-1$ соответствует изменению знака $\dot{H}$.

В модели, предложенной в работе [17] (см. также работы [14], [18], [20], [23], [24]), наша Вселенная рассматривается как медленно распадающаяся D3-брана, динамика которой задается тахионом открытой струны. Для описания динамики данного тахиона используется метод обрезания по уровням. Примечательной особенностью подобной динамики тахиона является нелокальное полиномиальное взаимодействие [19], [25]-[30]. Как показано в работе [31], тахион открытой струны эффективно моделируется скалярным полем с отрицательным кинетическим членом.

Обратная реакция браны описывается динамикой тахиона замкнутой струны. Скалярное поле $\xi$ связано с сектором замкнутых струн [32]. Его локальное описание содержит стандартный кинетический член [22] и, возможно, неполиномиальное самодействие [33]. 
В данной статье мы рассматриваем локальные модели с эффективными полиномиальными потенциалами $V(\phi, \xi)$. Форма этих потенциалов предполагается заданной кубической теорией открытых струн с помощью метода обрезания по уровням [25], [26]. В случае плоского пространства-времени эффективная локальная теория обладает четным потенциалом четвертой степени и имеет решения типа кинка. В неплоском случае для сохранения аналитического вида решений нужно рассмотреть четный потенциал шестой степени, переходящий в пределе плоского пространства-времени в потенциал типа Хиггса [18]. Точная форма взаимодействия открытых и замкнутых струн неизвестна, поэтому, следуя статье [20], мы рассматриваем простейшее полиномиальное взаимодействие.

Мы налагаем на потенциал $V(\phi, \xi)$ следующие ограничения:

- потенциал - полином шестой степени:

$$
V(\phi, \xi)=\sum_{k=0}^{6} \sum_{j=0}^{6-k} c_{k j} \phi^{k} \xi^{j}
$$

- потенциал является четным: $V(\phi, \xi)=V(-\phi,-\xi)$;

- коэффициенты при 5 -й и 6 -й степенях имеют порядок $1 / m_{\mathrm{p}}^{2}$, и в пределе $m_{\mathrm{p}}^{2} \rightarrow \infty$ получается потенциал четвертой степени.

Из полевой теории струн мы также предположим асимптотические условия для полей. Напомним, что имеется в виду следующая картина. Мы предполагаем, что фантомное поле $\phi(t)$ плавно движется из нестабильного возмущенного вакуума $(\phi=0)$ в невозмущенный и останавливается в нем. Другими словами, функция $\phi(t)$ обращается в нуль в некоторой точке (пусть $\phi(0)=0)$ и стремится к ненулевой асимптотике при $t \rightarrow+\infty: \phi(+\infty)=A$. Поле $\xi(t)$ соответствует замкнутой струне и стремится к нулю при $t \rightarrow+\infty$.

\section{3. МЕТОД СУПЕРПОТЕНЦИАЛА}

Гравитационные модели со скалярными полями играют важную роль в космологии и теориях с дополнительными измерениями. Одной из главных проблем исследования подобных моделей является нахождение точных решений неинтегрируемых уравнений движения.

В то же время легко построить потенциал для заданных частных решений. Если явная форма полей $\phi(t)$ и $\xi(t)$ задана, то, используя уравнение $(3)$, мы получаем $H(t)$ с точностью до константы:

$$
H(t)=\frac{1}{2 m_{\mathrm{p}}^{2}}\left(\int^{t} \dot{\phi}^{2}(\tau) d \tau-\int^{t} \dot{\xi}^{2}(\tau) d \tau\right)+C .
$$

Потенциал как функцию времени можно выразить через $H(t)$ :

$$
V(t)=m_{\mathrm{p}}^{2}\left(3 H(t)^{2}+\dot{H}(t)\right) .
$$

Этот прямой метод вычисления потенциала для заданных решений очень эффективен при рассмотрении одно- и двухполевых моделей с потенциалами вида 
$V(\phi, \xi)=e^{\alpha \xi} V_{1}(\phi)$ и $V(\phi, \xi)=e^{\alpha \phi} V_{2}(\xi)$, где $\alpha-$ константа [13]. Для двухполевых моделей с полиномиальным потенциалом данный метод не столь эффективен. Отметим, что вышеупомянутый метод бесполезен при поиске новых решений уравнений движения, сответствующих построенному потенциалу.

В данной статье мы используем метод суперпотенциала и показываем, что этот метод позволяет найти не только потенциал, но и новые точные решения. Метод суперпотенциала был предложен для построения потенциалов, соответствующих точным решениям пятимерной гравитационной модели [34]. Главная идея метода заключается в рассмотрении функции $H(t)$ (параметра Хаббла в космологии) как функции (суперпотенциала) скалярных полей: $H(t)=W(\phi(t), \xi(t))$. Уравнение (3) можно переписать в виде

$$
\frac{\partial W}{\partial \phi} \dot{\phi}+\frac{\partial W}{\partial \xi} \dot{\xi}=\frac{1}{2 m_{\mathrm{p}}^{2}}\left(\dot{\phi}^{2}-\dot{\xi}^{2}\right) .
$$

Если найден такой суперпотенциал $W(\phi, \xi)$, что выполнены следующие соотношения:

$$
\begin{aligned}
\dot{\phi} & =2 m_{\mathrm{p}}^{2} \frac{\partial W}{\partial \phi}, \quad \dot{\xi}=-2 m_{\mathrm{p}}^{2} \frac{\partial W}{\partial \xi} \\
V & =3 m_{\mathrm{p}}^{2} W^{2}+2 m_{\mathrm{p}}^{4}\left(\left(\frac{\partial W}{\partial \phi}\right)^{2}-\left(\frac{\partial W}{\partial \xi}\right)^{2}\right)
\end{aligned}
$$

то соответствующие $\phi(t), \xi(t)$ и $H(t)$ являются решением системы $(2)-(4)$.

Метод суперпотенциала разделяет систему уравнений (2)-(4) на две части: систему (10), которая, как правило, интегрируема при заданном полиноме $W(\phi, \xi)$, и уравнение (11), которое не интегрируемо, если $V(\phi, \xi)$ - полином, но имеет частные решения в виде полиномов. Стандартный способ применения метода суперпотенциала не предполагает решения уравнения (11). Потенциал $V(\phi, \xi)$ строится по заданному $W(\phi, \xi)$.

В двухполевых моделях метод суперпотенциала способствует нахождению новых решений. Действительно, дифференциальные уравнения (10) формируют систему второго порядка. Если эта система интегрируема, то мы получаем двухпараметрическое множество решений. Фиксация явного вида полей $\phi(t)$ и $\xi(t)$ равносильна заданию однопараметрического множества решений. Метод суперпотенциала позволяет обобщить это множество решений до двухпараметрического множества. С другой стороны, с помощью этого метода можно строить различные формы потенциала, соответствующие одному и тому же однопараметрическому множеству решений.

Идея рассмотрения параметра Хаббла как функции скалярных полей и преобразования (2)-(4) в систему (10), (11) используется в формулировке Гамильтона-Якоби уравнений Фридмана [35], [36] (см. также книгу [37]) и не связана с теориями суперсимметрии и супергравитации. В то же время метод, основанный на идее использования для поиска точных частных решений системы $(10),(11)$ вместо исходных уравнений движения, активно применяется в двухмерных полевых моделях [38], [39] и супергравитации [40]. Уравнения (10) известны как уравнения Богомольного [41] 
(см. также работу [39]). Метод суперпотенциала является комбинацией и естественным расширением этих двух методов. Он активно используется в космологии [8], [20], [42], [43]. Отметим обобщения данного метода на уравнения движения, описывающие замкнутые и открытые фридмановские Вселенные [42], системы с холодной темной материей [43] и теорию Бранса-Дикке [44].

\section{4. РАЗЛИЧНЫЕ ПОТЕНЦИАЛЫ С ОДИНАКОВЫМИ РЕШЕНИЯМИ}

В этом разделе мы покажем, что метод суперпотенциала позволяет находить различные формы потенциала $V(\phi, \xi)$ для одних и тех же $\phi(t), \xi(t)$ и $H(t)$.

Используя асимптотические условия, предположим следующую явную форму полей:

$$
\phi(t)=A \operatorname{th}(\omega t), \quad \xi(t)=\frac{A \sqrt{2(1+b)}}{\operatorname{ch}(\omega t)},
$$

причем $A>0, \omega>0$ и $b>-1$. Отметим, что эти решения являются естественным обобщением решений типа кинка, полученных в однополевой фантомной модели [18].

Построим потенциал, соответствующий полям (12). Функции $\phi$ и $\xi$ - решения следующей системы дифференциальных уравнений:

$$
\dot{\phi}=A \omega-\frac{\omega}{A} \phi^{2}, \quad \dot{\xi}=\omega \xi \sqrt{1-\frac{\xi^{2}}{2(1+b) A^{2}}} .
$$

Прямое использование метода суперпотенциала дает

$$
\frac{\partial W}{\partial \phi}=\frac{\omega}{2 m_{\mathrm{p}}^{2}}\left(A-\frac{1}{A} \phi^{2}\right), \quad \frac{\partial W}{\partial \xi}=-\frac{\omega \xi}{2 m_{\mathrm{p}}^{2}} \sqrt{1-\frac{\xi^{2}}{2(1+b) A^{2}}}
$$

Следовательно,

$$
H \equiv W=\frac{\omega}{6 m_{\mathrm{p}}^{2}}\left(3 A \phi-\frac{\phi^{3}}{A}-\sqrt{\frac{\left(2(1+b) A^{2}-\xi^{2}\right)^{3}}{2(1+b) A^{2}}}+H_{0}\right),
$$

где $H_{0}$ - произвольная константа. Различные значения $H_{0}$ соответствуют различным $V(\phi, \xi)$. Полученные потенциалы

$$
V=\omega^{2}\left(\frac{\left(A^{2}-\phi^{2}\right)^{2}}{2 A}-\frac{\xi^{2}}{2}+\frac{\xi^{4}}{4(1+b) A^{2}}\right)+3 m_{\mathrm{p}}^{2} W^{2}
$$

полиномиальны только в случае плоского пространства-времени $\left(m_{\mathrm{p}}^{2}=\infty\right)$ и не удовлетворяют условиям, рассматриваемым в разделе 2. Чтобы построить полиномиальный потенциал, мы используем тот факт, что функции (12) удовлетворяют не только системе (13), но также и следующей системе уравнений:

$$
\dot{\phi}=A b \omega\left(\frac{\phi^{2}}{A^{2}}-1\right)+\frac{\omega \xi^{2}}{2 A}, \quad \dot{\xi}=-\frac{\omega}{A} \phi \xi .
$$

Соответствующие параметр Хаббла (суперпотенциал) и потенциал имеют вид (чтобы получить четный потенциал, мы положили $H_{0}=0$ )

$$
H=\widetilde{W}=\frac{\omega \phi}{2 m_{\mathrm{p}}^{2}}\left(A b\left(\frac{\phi^{2}}{3 A^{2}}-1\right)+\frac{\xi^{2}}{2 A}\right),
$$




$$
\widetilde{V}=\frac{\omega^{2}}{2}\left(b\left(\phi^{2}-1\right)+\frac{1}{2} \xi^{2}\right)^{2}-\frac{\omega^{2}}{2 A^{2}} \phi^{2} \xi^{2}+\frac{3 \omega^{2} \phi^{2}}{4 m_{\mathrm{p}}^{2}}\left(A b\left(\frac{\phi^{2}}{3 A^{2}}-1\right)+\frac{\xi^{2}}{2 A}\right)^{2} .
$$

Этот пример показывает, что одинаковые функции $\phi(t), \xi(t)$ и $H(t)$ могут соответствовать существенно различным потенциалам $V(\phi, \xi)$. Кроме того, решения не изменятся при добавлении к потенциалу $\widetilde{V}$ (или $V$ ) любой функции $\delta V$ такой, что $\delta V, \partial(\delta V) / \partial \phi$ и $\partial(\delta V) / \partial \xi$ равны нулю на решениях. Например,

$$
\delta V=K(\phi, \xi)\left[\phi^{2}+\frac{1}{2(1+b)} \xi^{2}-A^{2}\right]^{2},
$$

где $K(\phi, \xi)$ является гладкой функцией.

\section{5. ПОСТРОЕНИЕ РЕШЕНИЙ С ПОМОЩЬЮ МЕТОДА СУПЕРПОТЕНЦИАЛА}

В предыдущем разделе мы показали, как можно выбрать потенциал для заданных решений. Теперь мы продемонстрируем возможность нахождения новых решений с помощью метода суперпотенциала. Рассмотрим модель с потенциалом (19). Легко заметить, что система (17) имеет не только решения (12), но и тривиальные решения $\phi(t)= \pm A, \xi(t)=0$, а также решения

$$
\phi(t)=-A \operatorname{th}\left(\omega b\left(t-t_{0}\right)\right), \quad \xi(t)=0 .
$$

Если $\xi(t) \not \equiv 0$, то, используя второе уравнение системы (17), мы получаем дифференциальное уравнение второго порядка для $\xi(t)$ :

$$
\ddot{\xi}(t)=\omega^{2} b \xi(t)-\frac{\omega^{2} \xi^{3}(t)}{2 A^{2}}+\frac{(1-b) \dot{\xi}^{2}(t)}{\xi(t)} .
$$

Решения уравнения (22) получаются в квадратурах:

$$
t-t_{0}= \pm \int \frac{A \sqrt{2(1+b)} \xi^{b-1}}{\omega \sqrt{2 A^{2} \xi^{2 b}+2 A^{2} b \xi^{2 b}+\xi^{2 b+2}+2 A^{2} C+2 A^{2} b C}} d \xi,
$$

где $C$ и $t_{0}$ - произвольные константы. При некоторых значениях параметра $b$ решения системы (17) выписываются явно. Например, при $b=-1 / 2$ получаем

$$
\begin{aligned}
& \phi(t)=\frac{A\left(\left(C_{1}^{2} C_{2}^{2}+4 A^{2}\right) e^{\omega t}-C_{1}^{2} e^{-\omega t}\right)}{\left(C_{1}^{2} C_{2}^{2}+4 A^{2}\right) e^{\omega t}+2 C_{1}^{2} C_{2}+C_{1}^{2} e^{-\omega t}}, \\
& \xi(t)=\frac{4 C_{1} A^{2}}{\left(C_{1}^{2} C_{2}^{2}+4 A^{2}\right) e^{\omega t}+2 C_{1}^{2} C_{2}+C_{1}^{2} e^{-\omega t}},
\end{aligned}
$$

где $C_{1}$ и $C_{2}$ - произвольные параметры. Легко проверить, что при всех значениях $C_{1}$ и $C_{2}$, кроме $C_{1}=0$, решения $(24)$ и параметр Хаббла (18) имеют следующие асимптотики:

$$
\phi( \pm \infty)= \pm A, \quad \xi( \pm \infty)=0, \quad H(+\infty)=\frac{A^{2} \omega}{6 m_{\mathrm{p}}^{2}}
$$


Таким образом, построена гравитационная модель с двухпараметрическим множеством точных решений. Потенциал и решения удовлетворяют условиям, наложенным с помощью теории струн (см. раздел 2).

Проанализируем свойства полученных решений и космологические следствия. Система (17) инвариантна относительно замены $\xi(t)$ на $-\xi(t)$, таким образом, каждому решению $\phi(t)$ соответствуют два решения $\pm \xi(t)$. Отметим, что функция $\phi(t)$ инвариантна относительно замены $C_{1} \rightarrow-C_{1}$, тогда как $\xi(t)$ меняет знак. Параметр Хаббла зависит от $\xi^{2}$, поэтому без ограничения общности мы можем положить $C_{1}>0$.

Система (17) автономна, поэтому из существования решений вида $\{\tilde{\phi}(t), \tilde{\xi}(t)\}$ следует, что пара функций $\left\{\tilde{\phi}\left(t-t_{0}\right), \tilde{\xi}\left(t-t_{0}\right)\right\}$, где $t_{0} \in \mathbb{C}$, тоже должна быть решением. Удобно параметризовать решения (24) так, чтобы один из новых параметров соответствовал сдвигу по времени. Чтобы для действительных решений можно было наложить ограничение $t_{0} \in \mathbb{R}$, мы положим $C_{1}=e^{t_{0}}$, используя ограничение $C_{1}>0$. Для краткости записи положим $\omega=1$ и введем вместо $C_{2}$ параметр $C \equiv C_{1} C_{2}$. Решения (24) получаются в следующем виде:

$$
\begin{aligned}
& \phi(t)=\frac{A\left(\left(C^{2}+4 A^{2}\right) e^{t-t_{0}}-e^{-\left(t-t_{0}\right)}\right)}{\left(C^{2}+4 A^{2}\right) e^{t-t_{0}}+2 C+e^{-\left(t-t_{0}\right)}}, \\
& \xi(t)=\frac{4 A^{2}}{\left(C^{2}+4 A^{2}\right) e^{t-t_{0}}+2 C+e^{-\left(t-t_{0}\right)}} .
\end{aligned}
$$

Чтобы сравнить полученные решения с изначальными решениями (12), введем новый параметр: $t_{1}=t_{0}+t_{00}$, где

$$
t_{00} \equiv-\frac{1}{2} \ln \left(C^{2}+4 A^{2}\right) .
$$

Теперь функции $\phi(t)$ и $\xi(t)$ имеют вид

$$
\begin{aligned}
& \phi(t)=\frac{A\left(e^{t-t_{1}}-e^{-\left(t-t_{1}\right)}\right)}{e^{t-t_{1}}+2 C / \sqrt{C^{2}+4 A^{2}}+e^{-\left(t-t_{1}\right)}}, \\
& \xi(t)=\frac{4 A^{2}}{\sqrt{C^{2}+4 A^{2}}\left(e^{t-t_{1}}+2 C / \sqrt{C^{2}+4 A^{2}}+e^{-\left(t-t_{1}\right)}\right)} .
\end{aligned}
$$

Рассмотрим решения с $t_{1}=0$. Легко видеть, что в этом случае

$$
\phi(0)=0, \quad \dot{\phi}(0)=\frac{A \sqrt{C^{2}+4 A^{2}}}{C+\sqrt{C^{2}+4 A^{2}}}>0 \quad \text { и } \quad \dot{\xi}(0)=0 .
$$

Напомним, что мы используем условие $A>0$. Из формулы (3) следует, что $\dot{H}(0)>$ 0 , а из формулы (18) получаем, что $H(0)=0$. Таким образом, решения с $t_{1}=0$ и произвольным $C$ являются космологическими решениями типа баунс (bounce), иными словами, $a(t)$ имеет баунс в точке $t=0$ (о подобных решениях в космологических моделях с двумя скалярными полями см., например, [45]).

Рассмотрим зависимость поведения параметра Хаббла $H$ от значения параметpa $C$. В случае $C=0$ имеем решения

$$
\phi_{0}(t)=A \operatorname{th}\left(t-t_{1}\right), \quad \xi_{0}(t)=\frac{A}{\operatorname{ch}\left(t-t_{1}\right)} .
$$



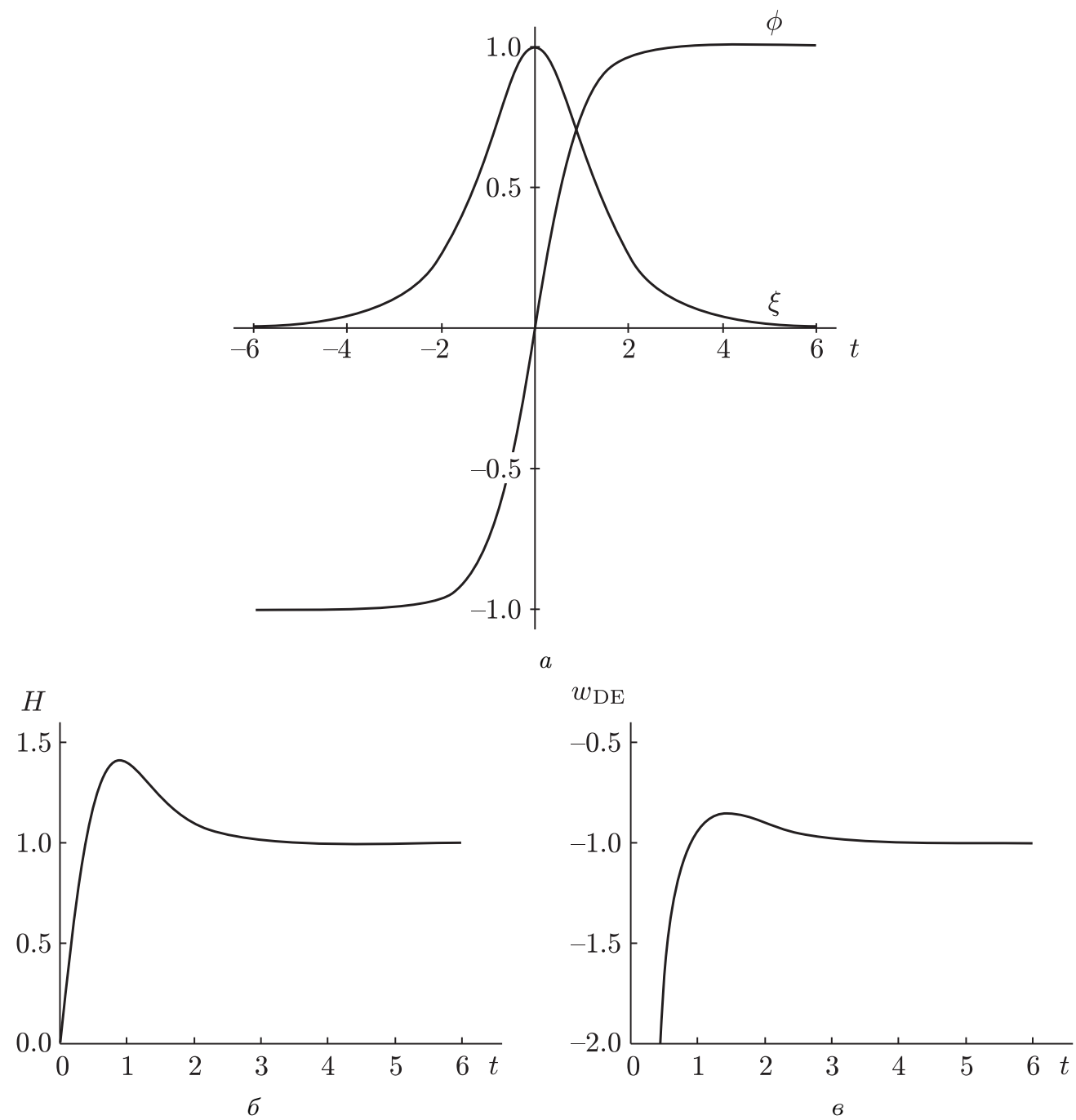

Рис. 1. Поля $\phi$ и $\xi(\mathrm{a})$, параметр Хаббла $H$ (б), параметр состояния $w_{\mathrm{DE}}$ при $C=0, t_{1}=0$ (в).

При $t_{1}=0$ мы получаем решения (12) с $\omega=1$. Соответствующий параметр Хаббла

$$
H_{0}=\frac{A^{2}}{6 m_{\mathrm{p}}^{2}}\left(3 \operatorname{th} t-2 \operatorname{th}^{3} t\right)
$$

имеет максимум в точке $t_{\max }=-\ln (\sqrt{2}-1) \simeq 0.881$ и убывает при $t \rightarrow \infty$. Поля $\phi_{0}$ и $\xi_{0}$, параметр Хаббла $H_{0}$ и параметр состояния $w_{\mathrm{DE}}$ представлены на рис. 1 (при построении рис. $1-3$ мы положили $A=1, \omega=1$ и $\left.m_{\mathrm{p}}^{2}=1 / 6\right)$. При произвольном $C$ 
параметр Хаббла имеет вид

$$
\begin{aligned}
H= & \frac{A^{2}\left(e^{t_{0}-t}+\left(C^{2}+4 A^{2}\right) e^{t-t_{0}}\right)^{2}}{6 m_{\mathrm{p}}^{2}\left(e^{t_{0}-t}+2 C+\left(C^{2}+4 A^{2}\right) e^{t-t_{0}}\right)^{3}}\left(e^{2\left(t_{0}-t\right)}+6 C e^{t_{0}-t}+\right. \\
& \left.+10\left(C^{2}+4 A^{2}\right)+6 C\left(C^{2}+4 A^{2}\right) e^{t-t_{0}}+\left(C^{2}+4 A^{2}\right)^{2} e^{2\left(t-t_{0}\right)}\right) .
\end{aligned}
$$

Прямые вычисления показывают, что для всех $C$, кроме $C= \pm 2 A, \dot{H}(t)=0$ в четырех точках:

$$
t_{\mathrm{m}_{k}}=t_{0}-\ln \left(-\frac{4 A^{2}+C^{2} \pm 2 A \sqrt{8 A^{2}+2 C^{2}}}{(C \pm 2 A)\left(C^{2}+4 A^{2}\right)}\right), \quad k=1, \ldots, 4,
$$

где два символа “ \pm ” независимы. Отметим, что при $C \neq \pm 2 A$ выполняется условие $\ddot{H}\left(t_{\mathrm{m}_{k}}\right) \neq 0$. Следовательно, в точках $t_{\mathrm{m}_{k}}$ параметр Хаббла $H(t)$ имеет экстремумы.

При $C>2 A$ ни одна из четырех точек $t_{\mathrm{m}_{k}}$ не принадлежит действительной оси.

Если $C=2 A$, то $\dot{H}(t)$ равняется нулю в двух точках, не принадлежащих действительной оси:

$$
\tilde{t}_{\mathrm{m}_{1}}=t_{0}-\ln (-2 A), \quad \tilde{t}_{\mathrm{m}_{2}}=t_{0}-\ln (-4 A) .
$$

Таким образом, при $C \geqslant 2 A$ параметр Хаббла $H(t)$ является монотонно возрастающей функцией, и его поведение напоминает поведение параметра Хаббла в однополевой фантомной модели [18].

При $-2 A<C<2 A$ функция $H(t)$ имеет экстремумы в двух точках. Если $t_{1}=0$, то $\phi(t)$ - нечетная функция, а $\xi(t)$ - четная. Следовательно, соответствующий параметр Хаббла, вычисленный с помощью формулы (18), является нечетной функцией. Легко проверить, что на полуоси $t>0$ параметр Хаббла $H(t)$ положителен и, следовательно, имеет максимум (см. рис. 2). Таким образом, поведение функции $H(t)$ при $-2 A<C<2 A$ похоже на ее поведение при $C=0$.

Если $C=-2 A$, то $\dot{H}(t)$ равняется нулю в двух точках:

$$
\tilde{t}_{\mathrm{m}_{3}}=t_{0}-\ln (2 A), \quad \tilde{t}_{\mathrm{m}_{4}}=t_{0}-\ln (4 A) .
$$

Легко проверить, что в этих точках $\ddot{H}= \pm 16 A^{2} / m_{\mathrm{p}}^{2} \neq 0$, следовательно, поведение параметра Хаббла качественно не отличается от приведенного на рис. 1 и 2.

Рассмотрим случай $C<-2 A$. Все четыре точки экстремума (33) действительны. Таким образом, выбрав $C<-2 A$, мы получаем качественно иное поведение параметра Хаббла.

Если $t_{1}=0$, то, как отмечалось выше, параметр Хаббла является нечетной функцией. Производная параметра Хаббла в нуле положительна, следовательно, $H(t)$ имеет максимум в некоторой точке $t_{\mathrm{m}_{1}}>0$, минимум в некоторой точке $t_{\mathrm{m}_{2}}>t_{\mathrm{m}_{1}}$ и монотонно возрастает при $t>t_{\mathrm{m}_{2}}$. Отметим, что $w_{\mathrm{DE}}<-1$ при $t>t_{\mathrm{m}_{2}}$. Таким образом, найдены точные решения, соответствующие немонотонной функции $H(t)$ с фантомным поведением при больших временах (см. рис. 3).

Итак, используя метод суперпотенциала, мы нашли для модели с потенциалом

$$
\widetilde{V}=\omega^{2}\left(\frac{1}{8}\left(1-\phi^{2}+\xi^{2}\right)^{2}-\frac{1}{2 A^{2}} \phi^{2} \xi^{2}+\frac{3 \phi^{2}}{4 m_{\mathrm{p}}^{2}}\left(\frac{A}{2}\left(1-\frac{\phi^{2}}{3 A^{2}}\right)+\frac{\xi^{2}}{2 A}\right)^{2}\right)
$$



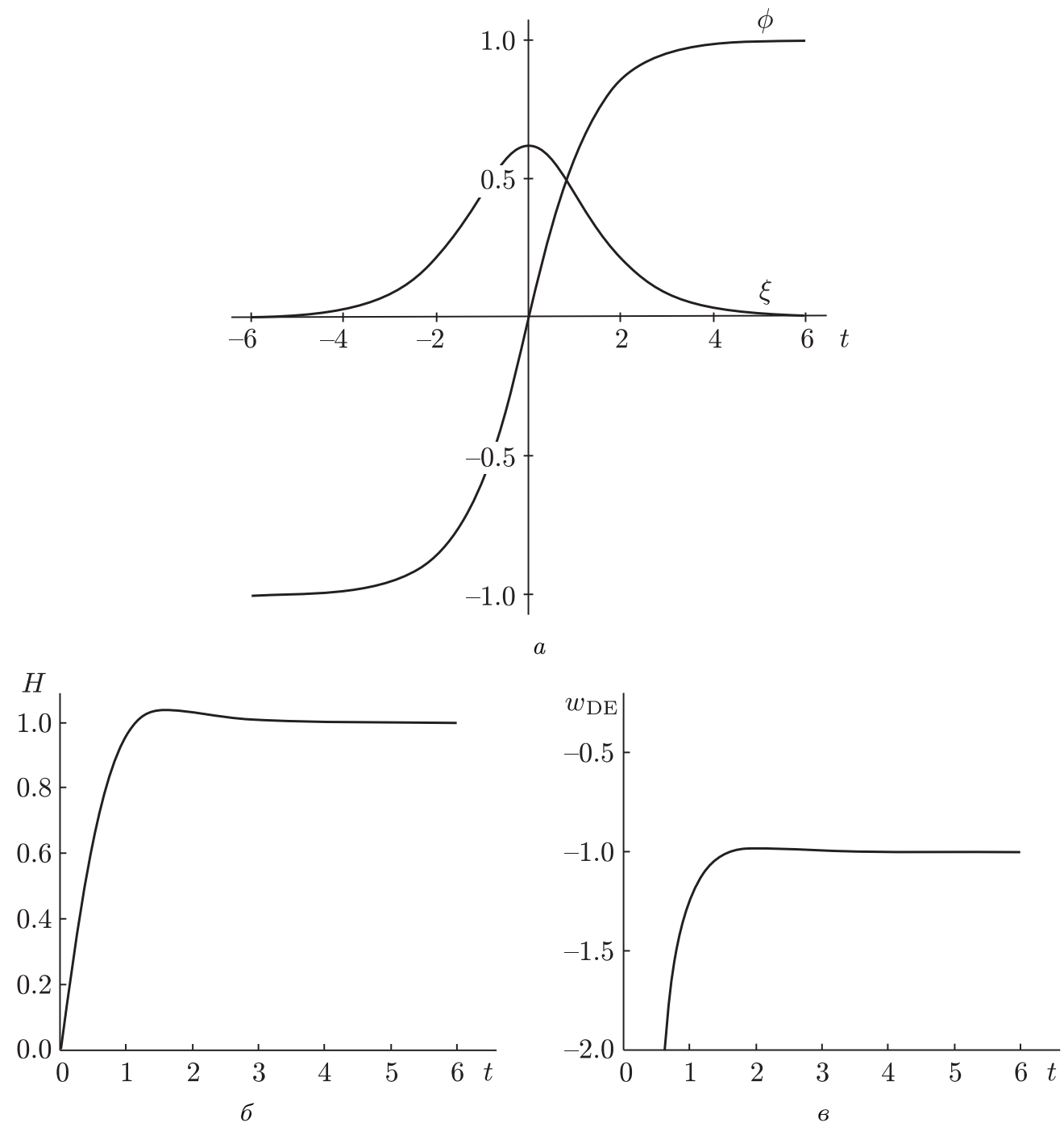

Рис. 2. Поля $\phi$ и $\xi(\mathrm{a})$, параметр Хаббла $H$ (б), параметр состояния $w_{\mathrm{DE}}$ при $C=1, t_{1}=0$ (в).

двухпараметрическое множество точных решений. Отметим, что полученные решения имеют одинаковые асимптотические условия, тогда как поведение параметра состояния $w_{\mathrm{DE}}$ при больших временах оказывается различным. Таким образом, можно сделать вывод о том, что связанная с теорией струн модель с полиномиальным потенциалом допускает как $w_{\mathrm{DE}}>-1$, так и $w_{\mathrm{DE}}<-1$ при больших временах. 

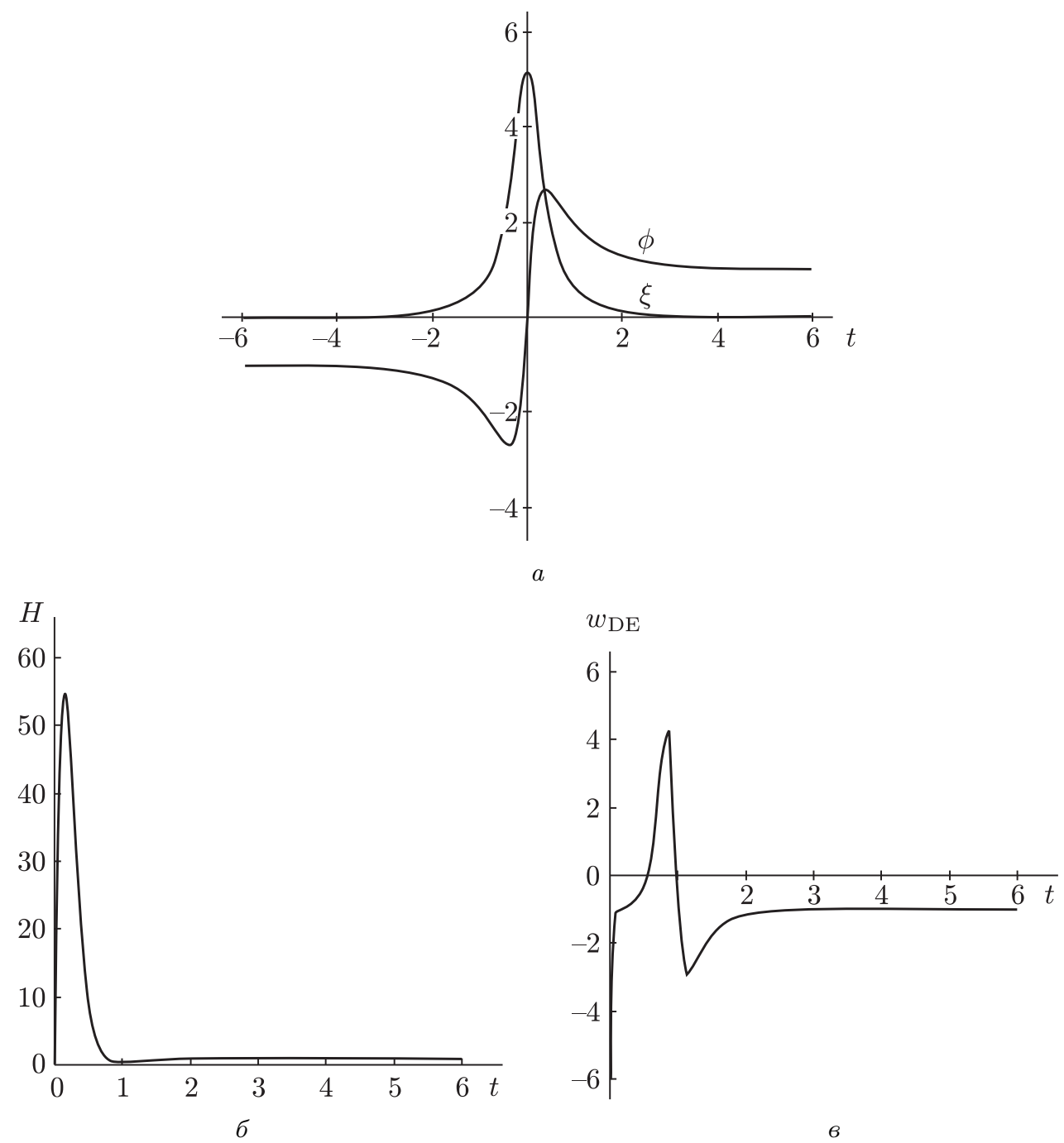

Рис. 3. Поля $\phi$ и $\xi(\mathrm{a})$, параметр Хаббла $H$ (б), параметр состояния $w_{\mathrm{DE}}$ при $C=-5, t_{1}=0$ (в).

\section{6. ЗАКЛЮЧЕНИЕ}

В данной статье мы исследовали динамику двухполевой модели темной энергии с одним фантомным полем и одним обычным скалярным полем. Построенная космологическая модель обладает полиномиальным потенциалом $V(\phi, \xi)$, происхождение которого связано с теорией струн. Найдено двухпараметрическое множество точных решений, которое может быть разделено на два подмножества таким образом, что одно подмножество соответствует при больших временах однополевым моделям квинтэссенции, а другое подмножество - однополевым фантомным моделям. Отме- 
тим, что оба подмножества полностью состоят из решений, удовлетворяющих одним и тем же асимптотическим условиям.

В статье мы активно используем метод суперпотенциала и показываем, что он позволяет не только строить потенциал для данного решения, но также и находить новые решения. Метод суперпотенциала позволяет расширить однопараметрическое множество решений до двухпараметрического. Использование метода суперпотенциала позволяет разделить исходную систему уравнений движения (2)-(4) на две части. Одна часть - уравнение для суперпотенциала (11), которое в общем случае неинтегрируемо, но для многих полиномиальных потенциалов имеет специальные решения в виде полинома. Подстановка этих решений в систему (10) дает систему однородных дифференциальных уравнений, обычно интегрируемых по крайней мере в квадратурах. Таким образом, метод суперпотенциала позволяет выделить из неинтегрируемой системы уравнений Фридмана интегрируемую подсистему. Отметим, что системы типа (10) активно исследуются в механике. С другой стороны, этот метод позволяет выбрать такую настройку параметров рассматриваемой гравитационной модели, (например, коэффициентов потенциала), что точные решения существуют в явном виде.

Для решения с $H(0)=0$ мы получаем $\dot{H}(0)>0$. Данные решения известны как баунс-решения (см. работу [45]). Отметим, что точные решения типа баунс могут быть получены и в нелокальных струнных космологических моделях [46].

Благодарности. Автор благодарит А.А. Андрианова, И.Я. Арефьеву и А. Ю. Каменщика за полезные обсуждения. Работа частично поддержана РФФИ (грант № 05-01-00758) и Программой поддержки ведущих научных школ (грант № НШ-8122.2006.2).

\section{Список литературы}

[1] A. Riess et al., Astron. J., 116:3 (1998), 1009-1038; arXiv: astro-ph/9805201.

[2] D. N. Spergel et al., Astrophys. J. Suppl. Ser., 148:1 (2003), 175-194; arXiv: astro-ph/0302209; Astrophys. J. Suppl. Ser., 170:2 (2007), 377-408; arXiv: astro-ph/0603449.

[3] M. Tegmark et al., Phys. Rev. D, 69:10 (2004), 103501; arXiv: astro-ph/0310723.

[4] P. Astier et al., Astron. Astrophys., 447:1 (2006), 31-48; arXiv: astro-ph/0510447.

[5] E. J. Copeland, M. Sami, Sh. Tsujikawa, Internat. J. Modern Phys. D, 15:11 (2006), 1753-1935; arXiv: hep-th/0603057.

[6] Y. Gong, A. Wang, Phys. Rev. D, 75 (2007), 043520; arXiv: astro-ph/0612196.

[7] U. Alam, V. Sahni, A. A. Starobinsky, J. Cosmol. Astropart. Phys., 2007, № 02, 011; arXiv: astro-ph/0612381.

[8] V. Sahni, A. A. Starobinsky, Internat. J. Modern Phys. D, 15:12 (2006), 2105-2132; arXiv: astro-ph/0610026.

[9] U. Alam, V. Sahni, T. D. Saina, A. A. Starobinsky, Mon. Not. R. Astron. Soc., 354:1 (2004), 275-291; arXiv: astro-ph/0311364.

[10] A. Vikman, Phys. Rev. D, 71:2 (2005), 023515; arXiv: astro-ph/0407107.

[11] Sh. Nojiri, S. D. Odintsov, J. Phys., 66:1 (2007), 012005; arXiv: hep-th/0611071; Phys. Lett. B, 639:3-4 (2006), 144-150; arXiv: hep-th/0606025; Gen. Relativ. Gravitation, 38:8 (2006), 1285-1304; arXiv: hep-th/0506212; Phys. Rev. D, 72:2 (2005), 023003; arXiv: hep-th/0505215. 
[12] Sh. Nojiri, S.D. Odintsov, Sh. Tsujikawa, Phys. Rev. D, 71:6 (2005), 063004; arXiv: hep-th/0501025; Sh. Tsujikawa, Phys. Rev. D, 72:8 (2005), 083512; arXiv: astro-ph/0508542; S. Nesseris, L. Perivolaropoulos, J. Cosmol. Astropart. Phys., 2007, № 01, 018; arXiv: astro-ph/0610092; R. Gannouji, D. Polarski, A. Ranquet, A. A. Starobinsky, J. Cosmol. Astropart. Phys., 2006, № 09, 016; arXiv: astro-ph/0606287; B. А. Рубаков, TMФ, 149:3 (2006), 409-426; arXiv: hep-th/0604153; H. Mohseni Sadjadi, M. Alimohammadi, Phys. Rev. D, 74:10 (2006), 103007; arXiv: gr-qc/0610080; M. Alimohammadi, H. Mohseni Sadjadi, Phys. Lett. B, 648:2-3 (2007), 113-118; arXiv: gr-qc/0608016; H. Mohseni Sadjadi, M. Alimohammadi, Phys. Rev. D, 74:4 (2006), 043506; arXiv: gr-qc/0605143; P.S. Apostolopoulos, N. Tetradis, Phys. Rev. D, 74:6 (2006), 064021; arXiv: hep-th/0604014; V. Sahni, Yu. Shtanov, J. Cosmol. Astropart. Phys., 2003, № 11, 014; arXiv: astro-ph/0202346; Wen Zhao, Yang Zhang, Phys. Rev. D, 73:12 (2006), 123509; arXiv: astro-ph/0604460; Bo Feng, Xiulian Wang, Xinmin Zhang, Phys. Lett. B, 607:1-2 (2005), 35-41; arXiv: astro-ph/0404224; Zong-Kuan Guo, Yun-Song Piao, Xinmin Zhang, Yuan-Zhong Zhang, Phys. Lett. B, 608:3-4 (2005), 177-182; arXiv: astro-ph/0410654; Xiao-Fei Zhang, Hong Li, Yun-Song Piao, Modern Phys. Lett. A, 21:3 (2006), 231-241; arXiv: astro-ph/0501652; Ming-zhe Li, Bo Feng, Xin-min Zhang, J. Cosmol. Astropart. Phys., 2005, № 12, 002; arXiv: hep-ph/0503268; Hrvoje Stefancic, Phys. Rev. D, 71:12 (2005), 124036; arXiv: astro-ph/0504518; Rong-Gen Cai, Hong-Sheng Zhang, Anzhong Wang, Commun. Theor. Phys., 44 (2005), 948; arXiv: hep-th/0505186; Yi-Fu Cai, Hong Li, Yun-Song Piao, Xinmin Zhang, Phys. Lett. B, 646:4 (2007), 141-144; arXiv: gr-qc/0609039; Xin Zhang, Phys. Rev. D, 74:10 (2006), 103505; arXiv: astro-ph/0609699; Hongsheng Zhang, Zong-Hong Zhu, Phys. Rev. D, 75:2 (2007), 023510; arXiv: astro-ph/0611834.

[13] A. A. Andrianov, F. Cannata, A. Yu. Kamenshchik, J. Phys. A, 39:32 (2006), 9975-9982; arXiv: gr-qc/0604126.

[14] I. Ya. Aref'eva, A. S. Koshelev, JHEP, 2007, № 02, 041; arXiv: hep-th/0605085.

[15] V.K. Onemli, R.P. Woodard, Class. Q. Grav., 19:17 (2002), 4607-4626; arXiv: gr-qc/0204065; S. M. Carroll, M. Hoffman, M. Trodden, Phys. Rev. D, 68:2 (2003), 023509; arXiv: astro-ph/0301273; St. D. H. Hsu, A. Jenkins, M. B. Wise, Phys. Lett. B, 597:3-4 (2004), 270-274; arXiv: astro-ph/0406043; R. V. Buniy, St. D. H. Hsu, B. M. Murray, Phys. Rev. D, 74:6 (2006), 063518; arXiv: hep-th/0606091; B. McInnes, Nucl. Phys. B, 718:1-2 (2005), 55-82; arXiv: hep-th/0502209; V. Gorini, A. Yu. Kamenshchik, U. Moschella, V. Pasquier, A. A. Starobinsky, Phys. Rev. D, 72:10 (2005), 103518; arXiv: astro-ph/0504576; E. O. Kahya, V. K. Onemli, Phys. Rev. D, 76:4 (2007), 043512; arXiv: gr-qc/0612026.

[16] I. Ya. Aref'eva, I. V. Volovich, On the null energy condition and cosmology, arXiv: hep-th/0612098.

[17] I. Ya. Aref'eva, "Nonlocal string tachyon as a model for cosmological dark energy", p-Adic Mathematical Physics: 2nd International Conference, AIP Conf. Proc., 826, eds. A. Yu. Khrennikov, Z. Rakic, I. V. Volovich, Amer. Inst. Phys., Melville, NY, 2006, 301-311; arXiv: astro-ph/0410443.

[18] И. Я. Арефьева, А. С. Кошелев, С. Ю. Вернов, ТМФ, 148:1 (2006), 23-41; arXiv: astro-ph/0412619.

[19] A. Sen, Internat. J. Modern Phys. A, 20:24 (2005), 5513-5656; arXiv: hep-th/0410103; $\mathrm{K}$. Ohmori, A review on tachyon condensation in open string field theories, arXiv: hep-th/0102085; I. Ya. Aref'eva, D. M. Belov, A. A. Giryavets, A.S. Koshelev, P. B. Medvedev, "Noncommutative field theories and (super)string field theories", Particles and Fields, Proc. 11th Jorge André Swieca summer school (São Paulo, Brazil, January 14-27, 2001), ed. A. V. Gilvan, World Scientific, Singapore, 2002, 1-164; arXiv: 
hep-th/0111208; W. Taylor, Lectures on D-branes, tachyon condensation an string field theory, arXiv: hep-th/0301094.

[20] I. Ya. Aref'eva, A. S. Koshelev, S. Yu. Vernov, Phys. Rev. D, 72:6 (2005), 064017; arXiv: astro-ph/0507067.

[21] I. Ya. Aref'eva, L. V. Joukovskaya, JHEP, 2005, № 10, 087; arXiv: hep-th/0504200.

[22] L. V. Joukovskaya, Ya. I. Volovich, Energy flow from open to closed strings in a toy model of rolling tachyon, arXiv: math-ph/0308034.

[23] I. Ya. Aref'eva, A. S. Koshelev, S. Yu. Vernov, Phys. Lett. B, 628:1-2 (2005), 1-10; arXiv: astro-ph/0505605.

[24] I. Ya. Aref'eva, A. S. Koshelev, S. Yu. Vernov, Bulg. J. Phys., 33:Suppl. 1 (2006), 360-367.

[25] I. Ya. Arefeva, D. M. Belov, A. S. Koshelev, P. B. Medvedev, Nucl. Phys. B, 638:1-2 (2002), 3-20; arXiv: hep-th/0011117.

[26] I. Ya. Arefeva, D. M. Belov, A. S. Koshelev, P. B. Medvedev, Nucl. Phys. B, 638:1-2 (2002), 21-40; arXiv: hep-th/0107197.

[27] E. Witten, Nucl. Phys. B, 268:2 (1986), 253-294; Nucl. Phys. B, 276:2 (1986), 291-324.

[28] I. Ya. Aref'eva, P. B. Medvedev, A. P. Zubarev, Phys. Lett. B, 240:3-4 (1990), 356-362; Nucl. Phys. B, 341:2 (1990), 464-498.

[29] C. R. Preitschopf, C. B. Thorn, S. A. Yost, Nucl. Phys. B, 337:2 (1990), 363-433.

[30] N. Berkovits, A. Sen, B. Zwiebach, Nucl. Phys. B, 587:1-3 (2000), 147-178; arXiv: hep-th/0002211.

[31] I. Ya. Aref'eva, L. V. Joukovskaya, A.S. Koshelev, JHEP, 2003, №9, 012; arXiv: hep-th/0301137; I. Ya. Aref'eva, Fortschr. Phys., 51:7-8 (2003), 652-657.

[32] K. Ohmori, Phys. Rev. D, 69:2 (2004), 026008; arXiv: hep-th/0306096.

[33] B. Zwiebach, Ann. Phys., 267:2 (1998), 193-248; arXiv: hep-th/9705241.

[34] O. DeWolfe, D.Z. Freedman, S. S. Gubser, A. Karch, Phys. Rev. D, 62:4 (2000), 046008; arXiv: hep-th/9909134.

[35] A. G. Muslimov, Class. Q. Grav., 7:2 (1990), 231-237.

[36] D. S. Salopek, J. R. Bond, Phys. Rev. D, 42:12 (1990), 3936-3962.

[37] A. R. Liddle, D. H. Lyth, Cosmological Inflation and Large-Scale Structure, Cambridge University Press, Cambridge, 2000.

[38] D. Bazeia, M. J. dos Santos, R. F. Ribeiro, Phys. Lett. A, 208:1-2 (1995), 84-88; arXiv: hep-th/0311265.

[39] D. Bazeia, F. A. Brito, Phys. Rev. D, 61:10 (2000), 105019; arXiv: hep-th/9912015.

[40] A. Brandhuber, K. Sfetsos, JHEP, 1999, № 10, 013; arXiv: hep-th/9908116.

[41] Е. Б. Богомольный, ЯФ, 24:4 (1976), 861-870.

[42] D. Bazeia, C. B. Gomes, L. Losano, R. Menezes, Phys. Lett. B, 633:4-5 (2006), 415-419; arXiv: astro-ph/0512197; D. Bazeia, L. Losano, J. J. Rodrigues, First-order formalism for scalar field in cosmology, arXiv: hep-th/0610028.

[43] D. Bazeia, L. Losano, J. J. Rodrigues, R. Rosenfeld, First-order formalism for dust, arXiv: astro-ph/0611770.

[44] A.S. Mikhailov, Yu. S. Mikhailov, M. N. Smolyakov, I. P. Volobuev, Class. Q. Grav., 24:1 (2007), 231-242; arXiv: hep-th/0602143.

[45] Yi-Fu Cai, Taotao Qiu, Yun-Song Piao, Mingzhe Li, Xinmin Zhang, JHEP, 2007, № 10, 071; arXiv: 0704.1090.

[46] I. Ya. Aref'eva, L. V. Joukovskaya, S. Yu. Vernov, JHEP, 2007, №07, 087; arXiv: hep-th/0701184; Dynamics in nonlocal linear models in the Friedmann-Robertson-Walker metric, arXiv: 0711.1364. 